\title{
Generalization of a Muscle-Reflex Control Model to 3D Walking*
}

\author{
Seungmoon Song and Hartmut Geyer
}

\begin{abstract}
The neural controller that generates human locomotion can currently not be measured directly, and researchers often resort to forward dynamic simulations of the human neuromuscular system to propose and test different controller architectures. However, most of these models are restricted to locomotion in the sagittal plane, which limits the ability to study and compare proposed neural controls for 3D-related motions. Here we generalize a previously identified reflex control model for sagittal plane walking to 3D locomotion. The generalization includes additional degrees of freedom at the hips in the lateral plane, their actuation and control by hip abductor and adductor muscles, and 3D compliant ground contact dynamics. The resulting 3D model of human locomotion generates normal walking while producing human-like ground reaction forces and moments, indicating that the proposed neural controller based on muscle reflexes generalizes well to $3 \mathrm{D}$ locomotion.
\end{abstract}

\section{INTRODUCTION}

The neural controller that generates locomotion behaviors in humans can currently not be measured directly. As a result, many researchers resort to forward dynamic simulations of the human neuromuscular system to propose and test different controller architectures [1]-[6]. These studies have demonstrated that different architectures can generate walking or running behaviors with more or less humanlike kinematics, kinetics and muscle activation patterns. Examples include models proposed based on the observation of central pattern generators (CPG) in neurophysiological studies [1]-[3], [6], [7]; on the equilibrium point hypothesis [4], [8]; and on muscle reflexes [5], [9]. However, among all the different models, only a few based on CPG control have been generalized to three-dimensional locomotion [3], which limits the ability to study and compare alternative neural controllers of 3D-related motions (including for instance body yaw and roll, and turning). Such a comparison would help to identify which of the proposed alternatives is more likely to govern human neural control.

Here we seek to generalize one of the alternative neural controllers to three-dimensional locomotion. In a previous study, we identified a sagittal plane model of walking control following the process of embedding principles of legged dynamics and control within muscle reflexes [5], [10]. We present the preliminary extension of this model into a threedimensional one. The model includes an extended mechanical system with additional degrees of freedom (DOFs) at the hips in the lateral plane, added hip abductor and adductor muscles (section II), and 3D compliant contact

\footnotetext{
*This work was in part supported by the NSF through the NSF ERC on Quality of Life Technology (EEC 0540865).

S. Song and $\mathrm{H}$. Geyer are with the Robotics Institute, Carnegie Mellon University, 5000 Forbes Avenue, Pittsburgh, PA 15213, USA. \{smsong, hgeyer\} at cs.cmu.edu
}

models (section III). We also extend the previous model's trunk balance control to include the lateral degrees of freedom (section IV). Our preliminary results show that the generalized 3D model successfully generates walking while producing human-like ground reaction forces (GRFs) and moments (GRMs) (section V). The results indicate that the proposed neural controller based on muscle reflexes well generalizes to 3D locomotion, and we discuss our plans to study with this model human neural control beyond steady walking (section VI).

\section{HUMAN MUSCULOSKELETAL MODEL}

\section{A. Musculoskeletal Model}

The 3D musculoskeletal system represents a human male with height $\mathrm{h}=180 \mathrm{~cm}$ and body mass $\mathrm{m}=80 \mathrm{~kg}$ by 7 segments connected by 8 internal DOFs, and driven by 18 muscletendon units (MTUs) (Fig. 1-a and b). The seven segments include trunk (which models the whole upper body), thighs, shanks and feet. These segments are connected by hip, knee and ankle revolute joints, where each hip joint has DOFs in both the sagittal and coronal planes; the remaining joints have DOFs only in the sagittal plane. The joints are actuated by nine Hill-type muscles per leg (Fig. 1-c, [11]). Seven muscles produce forces in the sagittal plane (soleus, SOL; tibialis anterior, TA; gastrocnemius, GAS; vastus, VAS; hamstring group, HAM; gluteus maximus, GLU; and grouped hip flexors, HFL) and the other two muscles produce forces in the lateral plane (grouped hip abductors, HAB; and grouped hip adductors, HAD). Each muscle generates a joint torque $\tau_{m, j}=F_{m} r_{m}\left(\varphi_{j}\right)$ at the joint $j$ it spans, where $r_{m}\left(\varphi_{j}\right)$ models constant moment arm $r_{0}$ for hip joints and variable moment arms $r_{0} \cos \left(\varphi-\varphi_{\max }\right)$ for knee joints.

The detailed values of the segments and muscles are listed in Tab. I and Tab. II, respectively. The segment parameters are the segment length $d_{S}$, the distance $d_{J}$ between the distal part of the segment and its proximal joint, the distance $d_{G}$ between the distal part and its center of mass (COM), the segment mass $m_{S}$, and the moments of inertia $\Theta$ around each principal axis. All length and distance parameters $\left(d_{S}\right.$, $d_{J}$ and $d_{G}$ ) are defined by three values $l, w$ and $h$ which correspond to the lengths in each principal axis (Fig. 1-a) (only non-zero values are shown in Tab. I). The muscle parameters are the maximum isometric force $F_{\max }$, the maximum contraction speed $v_{\max }$, the optimal fiber length $l_{\text {opt }}$, and the tendon slack length $l_{\text {slack }}$. The muscle attachment parameters are the moment arm $r_{0}$, the attachment reference angles $\varphi_{\max }$ and $\varphi_{\text {ref }}$, and the pennation angle coefficient $\rho$. All segment parameters and muscle parameters are estimated from reported physiological data [4], [12]-[14]. 
(a)

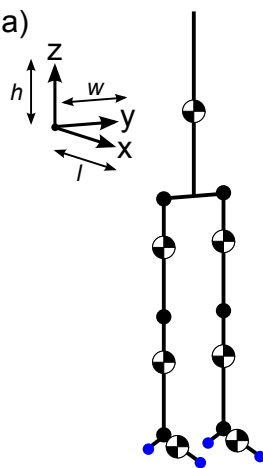

(b)

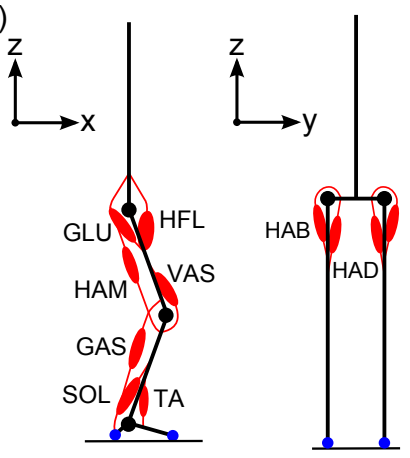

(c)
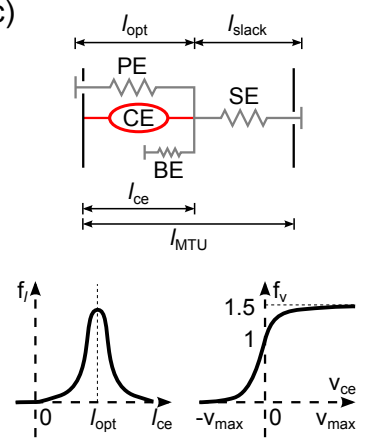

Fig. 1. Human musculoskeletal model. (a) The model consists of 7 segments, 8 DOF ( 2 at each hip), and 4 lumped compliant contact models (blue circles). (b) 9 muscle tendon units (MTUs) are attached to the skeletal system, 7 of which act in the sagittal plane while 2 act in the lateral (coronal) plane. (c) The MTU model consists of a contractile element (CE), parallel elastic element (PE), series elastic element (SE), and buffer elastic element (BE). The force-length and force-velocity relationships of the $\mathrm{CE}$ are sketched in the bottom.

\section{B. Contact Model}

The musculoskeletal model interacts with the ground via four lumped, compliant contact models. The contact models are located at the heel and ball of each foot, each computing GRFs in the normal and tangential directions and GRMs about the normal axis. To explain the implementation of the three dimensional contact models, we briefly cover the planar point contact model of [5] and then extend it to three dimensions.

In the planar point contact model, the vertical GRF is given by $F_{z}=k_{z} f_{c z}\left(\Delta z_{c}\right) f_{c \dot{z}}\left(\dot{z}_{c}\right)$, where $k_{z}$ is the stiffness coefficient, $\Delta z_{c}$ and $\dot{z}_{c}$ are ground penetration depth and velocity, and $f_{c z}$ and $f_{c \dot{z}}$ are piecewise linear functions (Fig. 2-a and b). The parameters $k_{z}$ and $\dot{z}_{c, M}$ of the functions $f_{c z}$ and $f_{c \dot{z}}$ define the contact stiffness and relaxation speed. We use $k_{z}=81.5 \mathrm{kNm}^{-1}$ and $\dot{z}_{c, M}=3 \mathrm{cms}^{-1}$. (Note that we also model joint limits similarly to the vertical point contact, exerting stopping torques instead of GRFs.) The horizontal GRF is modeled as either sliding or stiction friction (Fig. 2-a). A contact starts at sliding mode, switches to stiction mode if $\dot{x}_{c}<\dot{x}_{s t}=1 \mathrm{cms}^{-1}$, and switches back to sliding mode if $\left|F_{x, s t}\right|>\mu_{s t}\left|F_{z}\right|$, where $\mu_{s t}$ is set to 0.9 . Sliding friction acts opposite to the sliding direction $\dot{z}_{c}$ by $\left|F_{x, s l}\right|=\mu_{s l}\left|F_{z}\right|$, where $\mu_{s l}=0.8$, and the stiction friction is calculated in the same way as the vertical GRF assuming $k_{x}=8.2 \mathrm{kNm}^{-1}$ and $\dot{x}_{c, M}=3 \mathrm{cms}^{-1}$.

The point-contact model is extended to a three dimensional model by calculating the normal and tangential GRFs based

TABLE I

SEGMENT PARAMETERS.

\begin{tabular}{l|cccc} 
& foot & shank & thigh & trunk \\
\hline$d_{S}(\mathrm{~cm})$ & $20(1), 8(\mathrm{~h})$ & $46(\mathrm{~h})$ & $46(\mathrm{~h})$ & $80(\mathrm{~h}), 10(\mathrm{w})$ \\
$d_{J}(\mathrm{~cm})$ & $16(\mathrm{l}), 8(\mathrm{~h})$ & - & - & $20(\mathrm{w})$ \\
$d_{G}(\mathrm{~cm})$ & $13(\mathrm{l}), 6(\mathrm{~h})$ & $28(\mathrm{~h})$ & $28(\mathrm{~h})$ & $35(\mathrm{~h})$ \\
$m_{S}(\mathrm{~kg})$ & 1.25 & 3.5 & 8.5 & 53.5 \\
$\Theta_{x}\left(\mathrm{kgm}^{2}\right)$ & 0.0007 & 0.05 & 0.15 & 4.0 \\
$\Theta_{y}\left(\mathrm{kgm}^{2}\right)$ & 0.005 & 0.05 & 0.15 & 2.5 \\
$\Theta_{z}\left(\mathrm{kgm}^{2}\right)$ & 0.005 & 0.003 & 0.03 & 1.0
\end{tabular}

on the encountered surface slope. For instance, Fig. 2-c shows a rigid body with point contact models at each edge colliding with and sliding down a rough surface. To account for friction moments generated by multiple contact surfaces on the human foot, we lump two virtual point-contacts into one model (Fig. 2-d). The lumped contact models of the ball and heel mimic two point-contacts that are $10 \mathrm{~cm}$ and $5 \mathrm{~cm}$ apart in the lateral plane, assuming that the normal load is equally distributed between these two virtual point-contacts.

\section{Simulation Environment}

We implemented the musculoskeletal model in MatLab Simulink/SimMechanics (R2012a) using the ode15s solver.

\section{NEURAL CONTROLLER EXTENSION}

For 3D locomotion, we extend the previously developed muscle reflex controller in a simple way. The extension does not change any control for the sagittal plane muscles [5], but only focuses on the control of the muscles that actuate the lateral DOFs of the hips.

\section{A. Neural Control for Lateral Stability}

The control of the hip abductors (HAB) and adductors (HAD) is different between stance and swing. In stance, the

TABLE II

MUSCLE PARAMETERS.

SOL TA GAS VAS HAM GLU HFL HAB HAD

\begin{tabular}{l|ccccccccc}
\hline$F_{\max }(N)$ & 4000 & 800 & 1500 & 6000 & 3000 & 1500 & 2000 & 3000 & 4500 \\
$v_{\max }\left(l_{\text {opt }} s^{-1}\right)$ & 6 & 12 & 12 & 12 & 12 & 12 & 12 & 12 & 12 \\
$l_{\text {opt }}(\mathrm{cm})$ & 4 & 6 & 5 & 8 & 10 & 11 & 11 & 9 & 10 \\
$l_{\text {slack }}(\mathrm{cm})$ & 26 & 24 & 40 & 23 & 31 & 13 & 10 & 7 & 18 \\
$r_{0}(\mathrm{~cm})$ & 5 & 4 & $5(\mathrm{a})$ & 6 & $5(\mathrm{k})$ & 10 & 10 & 6 & 3 \\
& & & $5(\mathrm{k})$ & & $8(\mathrm{~h})$ & & & & \\
$\varphi_{\max }(\mathrm{deg})$ & 110 & 80 & $110(\mathrm{a})$ & 165 & $180(\mathrm{k})$ & - & - & - & - \\
& & & $140(\mathrm{k})$ & 16 & $-(\mathrm{h})$ & & & & \\
$\varphi_{\text {ref }}(\mathrm{deg})$ & 80 & 110 & $80(\mathrm{a})$ & 125 & $180(\mathrm{k})$ & 150 & 180 & 10 & 15 \\
& & & $165(\mathrm{k})$ & 125 & $155(\mathrm{~h})$ & & & & \\
$\rho$ & 0.5 & 0.7 & $0.7(\mathrm{a})$ & 0.7 & $0.7(\mathrm{k})$ & 0.5 & 0.5 & 0.7 & 1
\end{tabular}


(a)

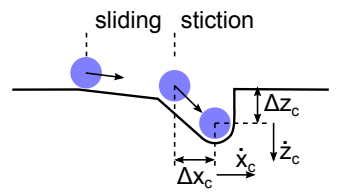

(b)

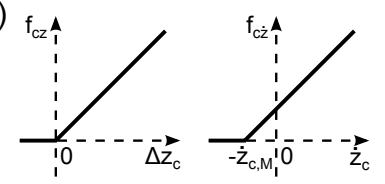

(d)

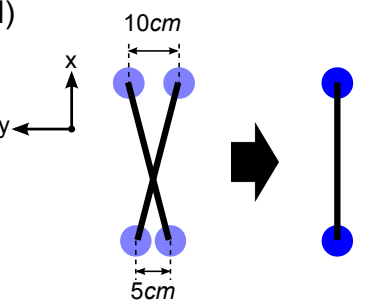

(c)

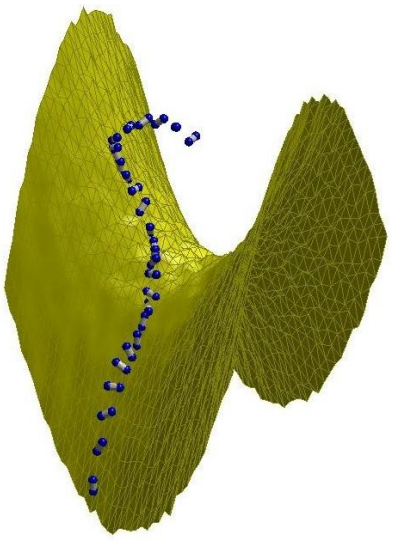

Fig. 2. Contact model. (a) A point-contact model (light blue circle) generates normal and horizontal GRFs. The horizontal GRF is generated assuming either sliding or stiction friction. (b) The normal GRF depends on the ground penetration $\Delta z_{c}$ and its velocity $\dot{z}_{c}$. (c) Demonstration of two $3 \mathrm{D}$ point-contact models attached to a rigid body colliding and sliding down a rough surface. (d) In the human model, two point-contact models representing lateral spread of the human foot at the ball and heel are lumped together in one model assuming the same normal force (blue circle). The virtual point-contact models of the ball and heel are $10 \mathrm{~cm}$ and $5 \mathrm{~cm}$ apart.

muscle stimulations are generated based on a desired torque that is required to stabilize the trunk in the upright position. Specifically, the desired torque $\tau_{H, L}^{d e s}$ of the hip muscles in the lateral plane has two components: a proportional-derivative (PD) feedback component and a feedforward compensation of the counter moment on the trunk induced by the contralateral swing leg. The components are weighted by the load the stance leg is bearing. The resulting desired torque is

$\tau_{H, L}^{\text {des }}=F_{\text {leg }}^{i p s i}\left(t_{s}\right)\left(-p_{H, L} \theta_{T, L}\left(t_{s}\right)-d_{H, L} \dot{\theta}_{T, L}\left(t_{s}\right)+\tau_{H, L}^{\text {contra }}\right)$

where $F_{\text {leg }}^{\text {ipsi }}$ is the load the leg is bearing, $p_{H, L}$ and $d_{H, L}$ are the PD gains, $\theta_{T, L}$ is the trunk's lateral lean angle with respect to the vertical axis, and $\tau_{H, L}^{\text {contra }}$ is the desired torque of the contralateral swing leg. The time $t_{s}$ indicates a delay of $5 \mathrm{~ms}$, which models the neural transmission delay.

In swing, the desired hip torque in the lateral direction is generated based on a desired angle

$$
\theta_{H, L}^{\text {des }}=c_{\theta 0}+c_{d} d_{C O M, L}+c_{v} v_{C O M, L},
$$

which predicts lateral foot placements required for maintaining gait stability [15]. Here, the parameters $c_{\theta 0}, c_{d}$ and $c_{v}$ are constant coefficients, and $d_{C O M, L}$ and $v_{C O M, L}$ are the lateral distance and velocity of the COM measured relative to the stance foot. The desired torque is calculated according to

$$
\tau_{H, L}^{\text {des }}=-p_{H, L}\left(\theta_{H, L}\left(t_{s}\right)-\theta_{H, L}^{\text {des }}\right)-d_{H, L} \dot{\theta}_{H, L}\left(t_{s}\right)
$$

where $\theta_{H, L}^{\text {des }}$ and $\dot{\theta}_{H, L}$ are the angle and angular velocity of the hip joint.

For both, stance and swing, the desired torque (eq. 1 and 3 ) is converted into muscle stimulation signals

$$
\begin{aligned}
& S_{H A B}=S_{0, H A B}+\tau_{H, L_{+}}^{\text {des }} /\left(F_{\text {max }, H A B} r_{0, H A B}\right) \\
& S_{H A D}=S_{0, H A D}+\tau_{H, L_{-}}^{\text {des }} /\left(F_{\text {max }, H A D} r_{0, H A D}\right)
\end{aligned}
$$

where $S_{0, i}$ is a prestimulation, $F_{\max }$ and $r_{0}$ are the maximum isometric forces and moment arms of each muscle, and the subscripts $+/$ - indicate positive/negative values $\left(\tau_{H, L}^{\text {des }}\right.$ is positive for abduction and negative for adduction). The factor $F_{\max , i} r_{0, i}$ scales the desired torque to muscle stimulation.

\section{B. Parameter Optimization for Walking}

To test if the proposing 3D neuromuscular system generates steady walking patterns, we use optimization on the control parameters. In particular, we use covariance matrix adaptation evolution strategy (CMA-ES) [16], a genetic algorithm for solving non-linear and non-convex optimization problems. We optimize over 48 parameters (all of the 30 sagittal plane and 9 lateral plane control parameters, and 9 initial conditions) to minimize the cost function

$$
J= \begin{cases}2 c_{L}-t_{\text {walk }}, & \text { fall } \\ c_{L}+d_{\text {steady }}, & \text { non-steady walk } \\ c_{1}\left|v_{\text {avg }}-v_{\text {tgt }}\right|+c_{2} C_{E}, & \text { steady walk }\end{cases}
$$

where $t_{\text {walk }}$ is the walking time until the model falls (if ever), $d_{\text {steady }}$ is a measure for steady walk, $v_{a v g}$ and $v_{t g t}$ are the average and target walking speeds, and $C_{E}$ is the energetic cost. $c_{1}, c_{2}$, and $c_{L}$ are empirically determined constants (10, 1, and 100, respectively). A step is assumed a steady step if the positions of every segments' edges do not change significantly relative to the touch-down position between subsequent heel-strikes. Specifically, we define $d_{\text {steady }} \leq$ $3 \mathrm{~cm}$ as a criterion for a steady step, where $d_{\text {steady }}$ is the sum of the differences of the relative positions of the edges. We assume the model to be in steady walking if the criterion is fulfilled for six consecutive steps (which proved to be a reliable indicator of steady walking in extensive tests). If a simulation achieves steady walking, the cost function is determined by the horizontal walking speed $v_{t g t}=\left[v_{x, t g t}, v_{y, t g t}\right]^{T}=[1.3,0]^{T} \mathrm{~ms}^{-1}$ and the energetic cost $C_{E}=E_{M} /(m d)$, where $E_{M}$ is the total metabolic energy consumed by all muscles estimated from a muscle energy model [17], $m$ is the body mass, and $d$ is the traveled distance.

\section{RESULTS}

The parameter optimization finds steady walking of the 3D neuromuscular model at a normal speed of $1.3 \mathrm{~ms}^{-1}$; moreover, the resulting patterns of the GRFs and the normal GRM are similar to those observed in human walking (Fig. 3$a$ and $b$ ). The vertical GRF (GRFz) shows the characteristic "double-hump" pattern, the progressional GRF (GRFx) switches from negative to positive values, the lateral GRF (GRFy) mostly pushes medially, and the vertical ground reaction moment switches from external to internal compensating 


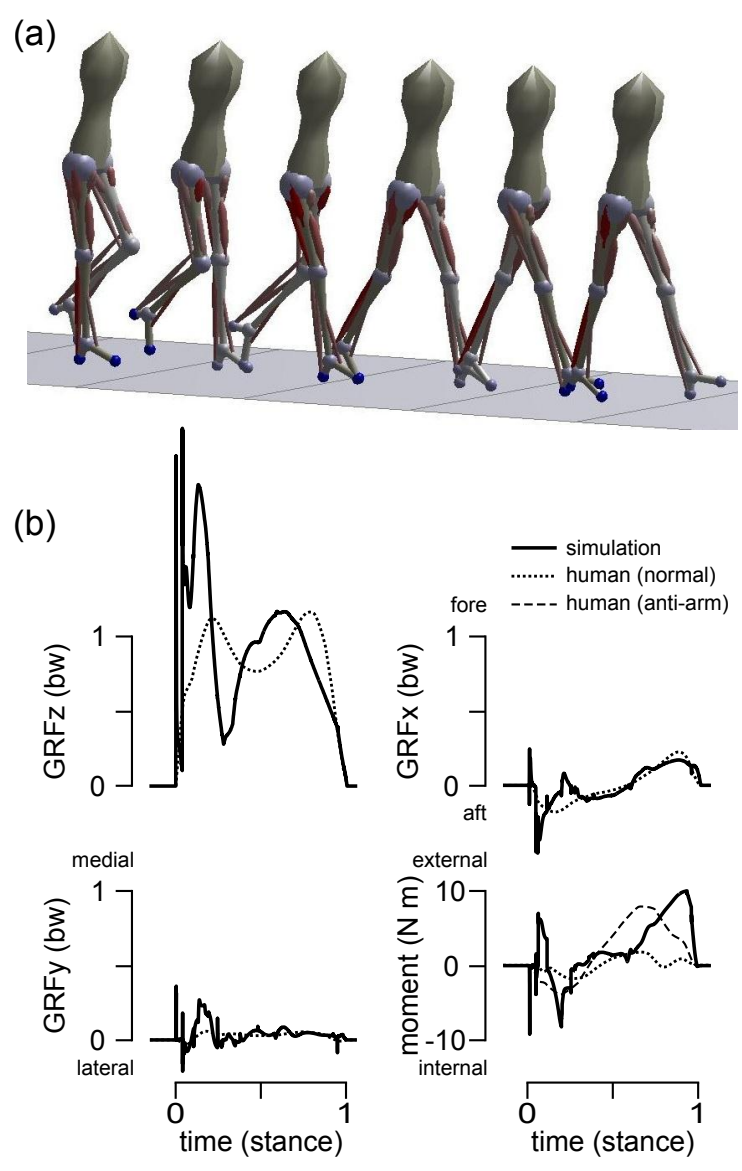

Fig. 3. Walking of the 3D neuromuscular human model. (a) The model walks at normal speed of $1.3 \mathrm{~ms}^{-1}$. Snapshots show the model at every $500 \mathrm{~ms}$, and lines on the ground are one meter apart. (b) The GRFs and GRM generated by the model (solid lines) qualitatively agree with the corresponding patterns of human walking (dotted lines, [18], [19]). The GRFs are normalized to body weight (bw). For the GRM, external rotation is defined as rotating the trunk counterclockwise/clockwise around the right/left stance leg.

for the angular momentum induced by swinging the leg [18], [19].

The model shows exaggerated GRF and GRM during the early stance phase, because it consists of rigid segments that generate high impact during heel strike. The magnitude of the GRM is comparable to that of human walking. While the magnitude of the GRM in humans is smaller during normal walking, it approaches the model-predicted value when swinging the arms in the opposite phase [19].

\section{CONCLUSION}

The 3D model successfully generates walking while producing human-like ground reaction forces (GRFs) and moments (GRMs). The results indicate that the proposed neural controller based on muscle reflexes generalizes well to $3 \mathrm{D}$ locomotion, opening up the opportunity to study human neural control beyond steady walking with this model. We plan to further detail the comparison to human walking by analyzing the joint kinematics, kinetics and muscle activation patterns, and to use this model to study 3D-relevant locomotion control.
On the other hand, the musculo-skeletal extensions that we used in the model were based on simplified assumptions about how the hip and foot are actuated in humans. For instance, we represented the hip motion with two decoupled DOFs which are actuated by separate muscles. However, in humans, the hip joints are spherical and hip muscles actuate multiple DOFs. We plan to investigate in simulation neural control strategies for these coupled joints and muscles with the goal of gaining insights into the functional advantages that these couplings provide.

\section{REFERENCES}

[1] G. Taga, Y. Yamaguchi, and H. Shimizu, "Self-organized control of bipedal locomotion by neural oscillators in unpredictable environment." Biol Cybern, vol. 65, no. 3, pp. 147-159, 1991.

[2] N. Ogihara and N. Yamazaki, "Generation of human bipedal locomotion by a bio-mimetic neuro-musculo-skeletal model." Biol Cybern, vol. 84 , no. 1, pp. 1-11, 2001.

[3] K. Hase, K. Miyashita, S. Ok, and Y. Arakawa, "Human gait simulationwith a neuromusculoskeletal model and evolutionarycomputation," J. Visual. Comput. Animat., vol. 14, pp. 73-92, 2003.

[4] M. Günther and H. Ruder, "Synthesis of two-dimensional human walking: a test of the $\lambda$-model," Biol. Cybern., vol. 89, pp. 89-106, 2003

[5] H. Geyer and H. Herr, "A muscle-reflex model that encodes principles of legged mechanics produces human walking dynamics and muscle activities," IEEE Trans Neural Syst Rehabil Eng, vol. 18, no. 3, 2010.

[6] Y. Kim, Y. Tagawa, G. Obinata, and K. Hase, "Robust control of cpg-based 3d neuromusculoskeletal walking model," Biological cybernetics, pp. 1-14, 2011.

[7] S. Grillner, "Locomotion in vertebrates: central mechanisms and relex interaction," Physiol. Rev., vol. 55, pp. 274-304, 1975.

[8] A. Feldman et al., "Once more on the equilibrium-point hypothesis (lambda model) for motor control." Journal of motor behavior, vol. 18 , no. 1 , p. $17,1986$.

[9] A. Prochazka, D. Gillard, and D. Bennett, "Positive force feedback control of muscles," Journal of neurophysiology, vol. 77, no. 6, pp. 3226-3236, 1997.

[10] S. Song and H. Geyer, "Regulating speed and generating large speed transitions in a neuromuscular human walking model," in Robotics and Automation (ICRA), 2012 IEEE International Conference on. IEEE, 2012, pp. 511-516.

[11] T. Hill et al., "Theoretical formalism for the sliding filament model of contraction of striated muscle. part i." Progress in biophysics and molecular biology, vol. 28, p. 267, 1974.

[12] R. Chandler, C. Clauser, J. McConville, H. Reynolds, and J. Young, "Investigation of inertial properties of the human body," DTIC Document, Tech. Rep., 1975.

[13] G. Yamaguchi, A. Sawa, D. Moran, M. Fessler, and J. Winters, "A survey of human musculotendon actuator parameters," Multiple muscle systems: Biomechanics and movement organization, pp. 717773, 1990.

[14] E. Arnold, S. Ward, L. R.L., and S. Delp, "A model of the lower limb for analysis of human movement," Annals of Biomedical Engineering, vol. 38, no. 2, pp. 269-279, 2010.

[15] K. Yin, K. Loken, and M. van de Panne, "Simbicon: simple biped locomotion control," in ACM Transactions on Graphics (TOG), vol. 26, no. 3. ACM, 2007, p. 105.

[16] N. Hansen, "The cma evolution strategy: A comparing review," Towards a New Evolutionary Computation. Advances on Estimation of Distribution Algorithms. Springer, p. 75.102, 2006.

[17] B. R. Umberger, K. G. Gerritsen, and P. E. Martin, "A model of human muscle energy expenditure," Comp. Meth. Biomech. Biomedic. Eng., vol. 6, no. 2, pp. 99-111, 2003.

[18] M. Damavandi, P. C. Dixon, and D. J. Pearsall, "Ground reaction force adaptations during cross-slope walking and running," Human movement science, vol. 31, no. 1, pp. 182-189, 2012.

[19] S. Collins, P. Adamczyk, and A. Kuo, "Dynamic arm swinging in human walking," Proceedings of the Royal Society B: Biological Sciences, vol. 276, no. 1673, pp. 3679-3688, 2009. 